\title{
News of BBS
}

Collaboration: BBS has established an Academic Scholarly Exchange and Collaborative Agreement with American University of Sovereign Nation (AUSN), Arizona, USA to promote cooperation and scholarly advancements in the fields of education, academic research, bioethics, disaster prevention and mitigation, global studies, public health and medicine, and peace. As a first initiative, AUSN took some BBS's Member for one year MBGPH (Master of Bioethics and Global Public Health) hybrid online and onsite master's degree program. AUSN provides scholarship to cover tuition.

BBS has also established collaboration with College of Medicine, National Taiwan University \& Hospital to build collaborative Research Platform and Professional Training Workshop for Cancer Treatment, Hospice/Palliative Care and Bioethics in Southeast Asian Countries.

Intensive Training Workshop: BBS is going to organize three days Intensive Training Workshop on Bioethics and Research: Preparing Ethical Professionals to be held on Dhaka, Bangladesh, 2-4 January 2015, at Centre for Advanced Research in Sciences, Dhaka University in collaboration between BBS and Eubios Ethics Institute and AUSN. All are requested to attend the workshop.

Speaker: Professor Darryl Macer, Director of the Institute of Indigenous Peoples and Global Studies, and Dean, Masters Program in Bioethics and Global Public Health (MBGPH), AUSN, USA.

Members of BBS in Conferences in Abroad: A team of two members attended the 15th Asian Bioethics Conference, 1- 9, November 2014 in Japan. They contributed with their scholarly research paper in the conference and enhance the prestige of BBS. Following papers were presented during the conference.

Public responsibility in healthcare. Shamima Parvin Lasker Professor \& Head of Anatomy, City Dental College, Dhaka, Bangladesh; General Secretary, Bangladesh Bioethics Society; Vice President for South Asia, Asian Bioethics Association.

Ethical issues in biomedical research on human subjects in developing countries, Dr. Abdus Shakoor, Associate Professor of Physical Medicine, BSMMU. 